Pesq. Vet. Bras. 29(10):816-822, outubro 2009

\title{
Avaliação dos parâmetros cardiorrespiratórios, hemogasométricos e da concentração plasmática de cortisol em bovinos submetidos à imobilização eletromagnética ${ }^{1}$
}

\author{
Ademir C. Rosa ${ }^{*}$, Aury N. Moraes ${ }^{3}$, Suzane L. Beier ${ }^{3}$, Nilson Oleskovicz ${ }^{3}$, \\ Doughlas Regalin ${ }^{2}$, Ruiney Carneiro ${ }^{4}$ e Corinne A.F. Nascimento ${ }^{4}$
}

\begin{abstract}
Rosa A.C., Moraes A.N., Beier S.L., Oleskovicz N., Regalin D., Carneiro R. \& Nascimento C.A.F. 2009. [Evaluation of the cardiorrespiratory and hemogasometrics effects and cortisol plasmatic concentration in bovine submitted of electromagnetic immobilization.] Avaliação dos parâmetros cardiorrespiratórios, hemogasométricos e da concentração plasmática de cortisol em bovinos submetidos à imobilização eletromagnética. Pesquisa Veterinária Brasileira 29(10):816-822. Departamento de Clínica e Cirurgia, Universidade do Estado de Santa Catarina, Lages, SC 88520-000, Brazil. E-mail: cassivetr@ hotmail.com

The ways of immobilization generally used in cattle provide excessive manipulation and stress for the animal. The pain is an emotional experience and unpleasant sensation in combination with tissue damage. The new method of physical restraint for cattle uses low intensity electromagnetic waves, promising immobilization without stress or painful stimulation for the animals. The aim of this study is to evaluate of cardiorespiratory effects and plasmatic cortisol concentration of cattle submitted to electromagnetic immobilization. Six healthy cows with average weight of $300.3 \pm 85.76 \mathrm{~kg}$ (mean \pm SD) were assigned to two treatments in a randomized design with a 15-day interval between experiments GC (control group) and GI (immobilization group). Data are presented as mean + SD. The statistical analyses used the t-Test between groups, and ANOVA random block design between times into same group. All statistical analyses were performed using $P<0.05$ as a significant level. Evaluation moments: M1 (basal); M1: after withdrawal probe, M2 and M3 (30 and 60 minutes after M1 respectively). There was a significant increase of FC and $f$ in M1, PAM in the moments (M1, M2, M3) and in the levels of plasmatic cortisol concentration in M1 e M2 in the immobilization group when to compared with the control group. It could be concluded that this electromagnetic immobilization is a method of physic restraint only, offers no anesthetic support and favors comfort and animal welfare.
\end{abstract}

INDEX TERMS: Pain, analgesia, stress, restraint.

${ }^{1}$ Recebido em 18 de fevereiro de 2009.

Aceito em 30 de junho de 2009.

2 Programa de Pós-Graduação, Mestrado em Ciência Animal, Área de concentração Anestesiologia Veterinária, Centro de Ciências Agroveterinárias (CAV), Universidade do Estado de Santa Catarina (UDESC), Av. Luiz de Camões 2090, Conta Dinheiro, Lages, SC 88520-000, Brasil. *Autor para correspondência: cassivetr@ hotmail.com

${ }^{3}$ Departamento de Clínica e Cirurgia, CAV-UDESC, Lages, SC.

${ }^{6}$ EMLA: Astrazeneca, Cotia, São Paulo.

${ }^{7}$ BD Angiocath: BD, São Paulo.

${ }^{8}$ BD PRN plug adaptador: BD, São Paulo.

${ }^{9}$ Rapidlabor 348, Bayer, São Paulo, SP.

10 IMOBOI, Korth-RFID, São Carlos, SP.
RESUMO.- As formas de imobilização em bovinos mais utilizadas levam a uma manipulação excessiva e consequentemente estressante para o animal. A dor é uma experiência emocional de sensação desagradável que vem associada a um prejuízo tecidual. Surge no mercado um método de contenção física para bovinos que propõe a utilização de um aparelho que funciona com ondas eletromagnéticas de baixa intensidade promovendo imobilização sem estresse e ou estímulo doloroso para o animal. O objetivo deste projeto foi avaliar os parâmetros cardiorrespiratórios e hemogasométricos bem como os níveis de cortisol de bovinos submetidos à imobilização 
eletromagnética, a fim de observar se tal método é eficaz na diminuição do estresse e/ou estímulo doloroso. Foram utilizados 6 bovinos, mestiços, peso médio de $300,3 \mathrm{~kg}$ $( \pm 85,76)$. Os animais foram alocados aleatoriamente em dois grupos (controle dele mesmo): grupo controle (GC), introdução da sonda via retal (desligada); grupo Imobilizador (Gl) introdução da sonda e permanência desta ligada por 5 minutos. Análise estatística utilizou o Teste T pareado entre os grupos $(P<0,05)$ e a avaliação entre tempos dentro de cada grupo análise de variância de uma única via com repetições múltiplas (ANOVA - RM). Momentos avaliados M0: basal; M1: imediatamente após a retirada da sonda; M2 e M3 (30 e 60 minutos após M1 respectivamente). Houve um aumento significativo nas variáveis: frequências cardíaca e respiratória em (M1), na Pressão arterial média (M1, M2, M3) e níveis de cortisol nos momentos $\mathrm{M} 1$ e $\mathrm{M} 2$ no grupo imobilizador quando comparado ao grupo controle. Com relação à resposta ao estímulo, o Gl teve resposta negativa no momento da estimulação, comparado ao GC (resposta máxima). De acordo com os resultados obtidos, podemos concluir que o imobilizador eletromagnético é um método, exclusivamente, de contenção física, não oferecendo nenhum suporte anestésico, para o conforto ou bem estar ao animal.

TERMOS DE INDEXAÇÃO: Dor, analgesia, estresse, contenção.

\section{INTRODUÇÃO}

A dor é uma experiência emocional de sensação desagradável que vem associada a um prejuízo tecidual (Wall 1992). A não ser que exista o reconhecimento de um estímulo nocivo pelo sistema nervoso central, nenhuma resposta ou adaptação irá ocorrer (Paddleford 2001). Médicos veterinários devem se conscientizar de que a dor é um fator biológico ativo, que pode causar todas as consequências perniciosas do estresse, como imunossupressão, retardo na cicatrização de feridas, aceleração de processos patológicos, entre outros, e, portanto, tem o dever moral e ético de reconhecer e tratar a dor nos animais (Fantoni \& Mastrocinque 2002). Portanto, as respostas fisiológicas ou comportamentais devem ser interpretadas para a avaliação da dor nos animais (Paddleford 2001). Os animais sentem dor, mas sua expressão de dor não é verbal.

Os processos dolorosos acarretam uma série de alterações fisiológicas que podem ser gravemente deletérias. A dor causa várias interferências nos eixos neuroendócrinos como aumento nos níveis de aldosterona (causando retenção de sódio e desbalanço hidroeletrolítico), cortisol e catecolaminas (responsáveis por alterações cardíacas como arritmias e aumento no consumo de oxigênio pelo miocárdio) (Fantoni \& Mastrocinque 2002). A pituitária, tireóide, paratireóide e glândula adrenal lançam uma cascata de hormônios na circulação sanguínea após uma injúria tecidual (Wall 1992).

Os procedimentos realizados nos animais de produção, como castrações, marcações e pequenas cirurgias, são realizados na maioria das vezes apenas com a contenção do animal, sem qualquer tipo de analgésico ou anestésico local, causando dor intensa (Sneddon \& Gentle 2001). Esses procedimentos desencadeiam no animal uma situação estressante e dolorosa, que pode acarretar em perda de peso, predispor os animais a doenças, atraso no crescimento, prejuízos reprodutivos, e outras alterações fisiológicas significantes (Moberg 1996). As formas mais utilizadas para a imobilização física de bovinos, quando da inspeção diagnóstica e/ou tratamento medicamentoso/profilático, são através de cordas e bretes de contenção trazendo manipulação excessiva dos animais, predispondo a fraturas e ferimentos e desencadeando um processo de estresse (Stöber 1990).

Surge no mercado uma forma de contenção física para bovinos, que promete imobilização sem estresse ao animal, sem estímulo doloroso e com segurança para a equipe que realiza o procedimento.

O aparelho testado funciona com ondas eletromagnéticas de baixa intensidade que permitem a imobilização suave e segura do animal sem nenhum dano físico ao seu organismo e sem nenhum efeito colateral (Korth-Rfid 2009).

Os autores tem como objetivos através do presente trabalho, avaliar o estresse produzido pela utilização do imobilizador eletromagnético via retal, e se o mesmo promove alguma forma de anestesia e/ou analgesia, por meio de avaliação dos níveis de cortisol, e mensuração de parâmetros como frequência cardíaca, respiratória, pressão arterial média.

\section{MATERIAL E MÉTODOS}

Este estudo foi submetido à análise e julgamento da Comissão de Ética em Experimentação Animal do Centro de Ciências Agroveterinárias (CAV), UDESC, sendo aprovado sem restrições, sob o protocolo 1.17/06.

Animais. Foram utilizados seis bovinos adultos mestiços (fêmeas) com peso de $300,3 \mathrm{Kg}( \pm 85,76)$ provenientes do HCV (Hospital de Clínica Veterinária), CAV-UDESC, Lages, SC. Cada animal foi submetido a exame físico e hemograma completo. $O$ experimento foi realizado somente no período da manhã, evitando assim o efeito do ciclo circadiano sobre os níveis de cortisol. Os animais passaram por um período de um mês para adaptação ao local de realização do experimento. Foram mantidos em piquetes com pastagem e água ad libitum e manejados sempre pela mesma equipe.

Delineamento experimental. No dia do experimento os animais permaneceram 30 minutos no tronco de contenção física para a adaptação, nesse período foi colocada uma pomada com anestésico local $^{6}$ na região dorsal da orelha, (tricotomia da face dorsal da orelha foi realizada no dia anterior ao experimento). Feita a anti-sepsia local, foi introduzido um catéter de polietileno $22 \mathrm{G}^{7}(5 \mathrm{~cm})$ acoplado ao adaptador $\mathrm{PRN}^{8}$ na artéria marginal para avaliação da PAM (pressão arterial média), e para a colheita de amostras para gasometria arterial e dosagem do cortisol plasmático (COR). Realizada a fixação do catéter com cola e esparadrapo, o mesmo foi preenchido com solução salina heparinizada $(5 \mathrm{Ul} / \mathrm{mL})$. Após a colocação do catéter os animais permaneceram em repouso por um período de intervalo de 15 minutos antes do início do experimento. 
Parâmetros avaliados. Os parâmetros estudados foram: frequência cardíaca ( $F C$ ) batimentos/minuto, obtida por auscultação cardíaca com estetoscópio; temperatura corporal interna $\left(\mathrm{T}^{\circ} \mathrm{C}\right)$ em graus Celsius por termômetro digital posicionado no reto dos animais; frequência respiratória $(f)$ movimentos/ minuto obtida por observação do movimento costo-abdominal; PAM avaliada através da utilização de um manômetro aneróide; gasometria arterial ${ }^{9}$ onde foram avaliados hematócrito (HTC), pressão parcial de oxigênio $\left(\mathrm{PaO}_{2}\right)$, pressão parcial de dióxido de carbono $\left(\mathrm{PaCO}_{2}\right)$, potencial hidrogeniônico $(\mathrm{pH})$, níveis de sódio $\left(\mathrm{Na}^{+}\right)$, potássio $\left(\mathrm{K}^{+}\right)$, bicarbonato $\left(\mathrm{HCO}_{3}^{-}\right)$, excesso de bases $(\mathrm{BE})$ e saturação de oxigênio na hemoglobina $\left(\mathrm{SatO}_{2}\right)$, através de amostras sanguíneas colhidas com seringas previamente heparinizadas.

Para análise do cortisol, foram colhidas amostras de sangue arterial $(5 \mathrm{ml})$ em tubos com anticoagulante (EDTA), essas amostras foram centrifugadas a $3000 \mathrm{rpm}$ por 10 minutos e o plasma congelado a $-20^{\circ} \mathrm{C}$. As dosagens foram realizadas no laboratório de reprodução animal da Faculdade de Medicina Veterinária e Zootecnia (FMVZ), Unesp Campus de Botucatu.

Resposta ao estímulo. A resposta ao estímulo doloroso foi obtida através de pinçamento da vulva em uma escala préestabelecida: zero (0) não responde ao estimulo doloroso; um (1) resposta fraca ao estimulo doloroso; dois (2) resposta moderada ao estímulo doloroso e três (3) resposta máxima ao estímulo doloroso. (Modificado da escala única descritiva (SDS) Holton et al. 1998).

Os animais foram distribuídos, randomicamente, em dois grupos com intervalo de uma semana entre os grupos. No Grupo Controle (GC), a sonda ${ }^{10}$ foi introduzida por via retal, permanecendo no corpo do animal, desligada, por 5 minutos. No Grupo Imobilizador (GI) a sonda permaneceu, pelos mesmos 5 minutos, porém ligada e, portanto, sob ação de ondas eletromagnéticas. Após esse período de 5 minutos, a sonda foi removida.

Os momentos de avaliação dos parâmetros relacionados foram:

Momento basal (M0), avaliação feita após o período mínimo de 15 minutos da instrumentação, com obtenção dos valores basais e posterior introdução da sonda imobilizadora; Momento imediatamente após a retirada da sonda imobilizadora (M1), e momentos M2 e M3, respectivamente, 30 e 60 minutos após a retirada da sonda imobilizadora.

Nos animais do grupo imobilizador, após a introdução da sonda desligada, essa era ligada na potência zero e observase 0 acréscimo nessa potência até que 0 animal curvasse a coluna (Korth-Rfid 2009), permanecendo ligada por 5 minutos nessa mesma potência.

Análise estatística. Foi realizada análise de variância (ANOVA) com repetições múltiplas $(P<0,05)$ seguida pelo teste Student Newman Keuls para comparação de momentos dentro de cada grupo, e para comparação entre grupos, realizado teste $T$ pareado $(P<0,05)$. Para análise de resposta ao estímulo doloroso, foi realizada análise descritiva, e para a comparação entre momentos dentro do mesmo grupo, e entre grupos realizamos o teste de Wilcoxon $(\mathrm{P}<0,001)$.

Os dados obtidos foram expressos em tabelas sob a forma de médias e desvios-padrão das médias.

\section{RESULTADOS}

Nos animais do grupo imobilizador (GI), houve um aumento significativo nos parâmetros FC, PAM, e HTC, quan- do comparados os valores basais $(\mathrm{M} 0)$ e os valores logo após a utilização da sonda imobilizadora (M1). A $f$ apresentou um aumento significativo em GI durante todos os momentos avaliados (Quadro 1). Houve uma diferença significativa em relação aos níveis plasmáticos de cortisol, em Gl os níveis apresentaram-se aumentados em M1 até 30 minutos após a retirada da sonda eletromagnética (M2), quando comparados com os valores basais (M0) e também em relação ao grupo controle. Esses valores 1 hora após a utilização da sonda imobilizadora voltaram aos valores basais (Quadro 1). Nos dados hemogasométricos (Quadro 1), os valores de $\mathrm{pH}$ reduziram em M1 e M2, porém clinicamente irrelevante, pois se apresentavam dentro da normalidade. Já em relação a $\mathrm{PaCO}_{2}$, houve uma redução significativa em $\mathrm{M} 1$ e $\mathrm{M} 2$ em relação aos valores basais ( $\mathrm{M} 0$ ), e em relação ao grupo controle. Com relação ao $\mathrm{HCO}_{3}^{-}$houve uma redução em M1 e M2 (com valores próximos dos valores mínimos de referência) quando comparado com o basal (M0), obtendo valores próximos dos basais em M3. O mesmo ocorreu com o BE. Em relação ao $\mathrm{K}^{+}$, foi demonstrada alteração apenas entre M1 e o momento basal (M0), sendo os demais momentos semelhantes aos valores basais. Os parâmetros $\mathrm{PaO}_{2}$, $\mathrm{Na}^{+}$e $\mathrm{SatO}_{2}$, não observaram-se alterações significativas quando comparadas com M0.

Quando comparado os grupos, houve um aumento significativo na FC e f em M1, no grupo Gl, nos demais tempos apesar de não observado diferenças estatísticas, os valores aferidos no Gl foram maiores que no GC. Na PAM, houve um aumento significativo em todos os momentos em Gl quando comparado ao GC. Os valores de HTC e $\mathrm{T}^{\circ} \mathrm{C}$ apresentaram-se semelhantes nos dois grupos, não apresentando diferença significativa (Quadro 1). Os valores de $\mathrm{pH}$ apresentaram-se estatisticamente menores no $\mathrm{M} 1$ do Gl. Foram observados que $\mathrm{O}_{\mathrm{HCO}^{-}}$e o BE apresentaram no momento $\mathrm{M} 1$, uma redução significativa em Gl quando comparado ao GC. Com relação a $\mathrm{PaCO}_{2}$, houve uma redução significativa em $\mathrm{M} 1$ e $\mathrm{M} 2$ no $\mathrm{Gl}$ em relação ao GC. Já os valores de $\mathrm{PaO}_{2}, \mathrm{Na}^{+}, \mathrm{K}^{+}$, $\mathrm{SatO}_{2}$, não apresentaram alterações significativas entre os grupos (Quadro 1).

Em relação à resposta ao estímulo doloroso, 100\% dos animais do GC, apresentaram resposta máxima ao estímulo doloroso nos momentos M0, M1 e M2 e em M3 $83,33 \%$ e $16,66 \%$ dos animais, apresentaram resposta máxima e moderada respectivamente ao estímulo doloroso. No Grupo imobilizador, $100 \%$ dos animais não apresentaram resposta ao estímulo doloroso em M1; em M2, 30 minutos após a utilização da sonda, $16,66 \%$ dos animais não apresentaram resposta ao estímulo doloroso; $33,32 \%, 16,66 \%$ e $33,32 \%$ dos animais apresentaram resposta fraca, moderada e máxima ao estímulo doloroso respectivamente. No momento M3 (1 hora após a retirada da sonda), $16,66 \%, 33,32 \%$ e $49,98 \%$ dos animais apresentaram resposta fraca, moderada e máxima ao estímulo doloroso respectivamente (Quadro 2). 
Quadro 1. Bovinos mestiços, fêmeas, segundo os valores médios de freqüência cardíaca (FC), em batimentos por minuto (bat/min); freqüência respiratória $(f)$, em movimentos por minuto (mov/min); temperatura retal (T), em graus Celsius (ㄷ); pressão arterial média (PAM), em milímetros de mercúrio ( $\mathrm{mmHg}$ ); hematócrito (HTC); cortisol plasmático (COR), em microgramas por decilítro $(\mu \mathrm{g} / \mathrm{dl})$; de potencial de íons hidrogênio $(\mathrm{pH})$; pressão parcial de dióxido de carbono $\left(\mathrm{PaCO}_{2}\right)$, em $\mathrm{mmHg}$; de pressão parcial de oxigênio $\left(\mathrm{PaO}_{2}\right)$, em $\mathrm{mmHg}$; concentração de íons $\mathrm{Na}^{+}, \mathrm{K}^{+}$, $\mathrm{HCO}_{3}{ }^{-}$, em milimoles por litro (mmol/L); excesso de bases (BE), em mmol/ $\mathrm{L}$, e saturação de oxigênio $\left(\mathrm{SatO}_{2}\right)$, em valores percentuais $(\%)$, do grupo controle (GC) e imobilizador (Gl), com as diferenças estatísticas entre grupos e entre cada momento (M0 após a introdução da sonda imobilizadora, e M1, M2 e M3, respectivamente, imediatamente, aos 30 e aos 60 minutos após a retirada da sonda) em cada grupo

\begin{tabular}{|c|c|c|c|c|c|}
\hline \multirow[t]{2}{*}{ Variáveis } & \multirow[t]{2}{*}{ Grupo } & \multicolumn{4}{|c|}{ Momentos } \\
\hline & & MO & M1 & M2 & M3 \\
\hline FC & $\mathrm{GC}$ & $65( \pm 6,74)$ & $68( \pm 7,59)$ & $68( \pm 11,02)$ & $65( \pm 9)$ \\
\hline (bat/min) & $\mathrm{GI}$ & $64( \pm 8)$ & $97( \pm 23)^{a b}$ & $83( \pm 16,7)$ & $75( \pm 23,1)$ \\
\hline$f$ & $\mathrm{GC}$ & $23( \pm 4,68)$ & $25( \pm 7,76)$ & $25( \pm 6,89)$ & $25( \pm 6,53)$ \\
\hline (mov/min) & $\mathrm{GI}$ & $23( \pm 4,13)$ & $33( \pm 4,84)^{\mathrm{ab}}$ & $29( \pm 7,34)^{b}$ & $30( \pm 5,51)^{b}$ \\
\hline \multirow[t]{2}{*}{$\mathrm{T}^{\circ} \mathrm{C}$} & GC & $38( \pm 0,74)$ & $37,4( \pm 0,75)^{b}$ & $38( \pm 0,7)$ & $38( \pm 0,57)$ \\
\hline & GI & $38( \pm 0,58)$ & $37,6( \pm 0,61)^{b}$ & $38( \pm 0,61)$ & $38( \pm 0,82)$ \\
\hline PAM & GC & $110( \pm 7,37)$ & $119( \pm 11,77)$ & $111( \pm 6,78)$ & $113( \pm 10,7)$ \\
\hline$(\mathrm{mmHg})$ & $\mathrm{Gl}$ & $113( \pm 6,4)$ & $158( \pm 9,4)^{a b}$ & $123( \pm 11)^{\mathrm{a}}$ & $122( \pm 8)^{\mathrm{a}}$ \\
\hline \multirow[t]{2}{*}{ HTC } & $\mathrm{GC}$ & $32( \pm 2,5)$ & $32( \pm 3,39)$ & $31( \pm 3,16)$ & $30( \pm 1,97)$ \\
\hline & $\mathrm{Gl}$ & $31( \pm 1,47)$ & $33( \pm 2,64)^{b}$ & $29( \pm 1,36)$ & $29( \pm 1,47)$ \\
\hline COR & $\mathrm{GC}$ & $3,7( \pm 1,99)$ & $3,5( \pm 2,1)$ & $3( \pm 2,15)$ & $3,2( \pm 1,53)$ \\
\hline$(\mu \mathrm{g} / \mathrm{dl})$ & GI & $4,3( \pm 2,39)$ & $6,9( \pm 3,8) a b$ & $7,6( \pm 4,47)^{a b}$ & $7,5( \pm 2,45)$ \\
\hline \multirow[t]{2}{*}{$\mathrm{pH}$} & GC & $7,459( \pm 0,02)$ & $7,471( \pm 0,02)$ & $7,461( \pm 0,02)$ & $7,453( \pm 0,03)$ \\
\hline & $\mathrm{GI}$ & $7,465( \pm 0,02)$ & $7,378( \pm 0,04)^{a b}$ & $7,425( \pm 0,04)^{b}$ & $7,461( \pm 0,02)$ \\
\hline \multirow{2}{*}{$\begin{array}{c}\mathrm{PaCO}_{2} \\
(\mathrm{mmHg})\end{array}$} & GC & $36( \pm 3,29)$ & $35( \pm 1,95)$ & $36( \pm 3,19)$ & $37( \pm 3,68)$ \\
\hline & $\mathrm{GI}$ & $35( \pm 3,4)$ & $32( \pm 2,78)^{a b}$ & $32( \pm 3,59)^{\mathrm{a} b}$ & $34( \pm 3,55)$ \\
\hline \multirow{2}{*}{$\begin{array}{c}\mathrm{PaO}_{2} \\
(\mathrm{mmHg})\end{array}$} & GC & $85( \pm 12,85)$ & $86( \pm 5,96)$ & $82( \pm 7,23)$ & $79( \pm 4,8)$ \\
\hline & GI & $86( \pm 8,95)$ & $93( \pm 7,83)$ & $89( \pm 7,18)$ & $99( \pm 7,18)$ \\
\hline \multirow[t]{2}{*}{$\mathrm{Na}^{+}$} & $\mathrm{GC}$ & $143( \pm 6,62)$ & $141( \pm 5,97)$ & $141( \pm 7,28)$ & $142( \pm 5,44)$ \\
\hline & GI & $143( \pm 5,58)$ & $147( \pm 4,49)$ & $147( \pm 3,5)$ & $144( \pm 3,03)$ \\
\hline \multirow[t]{2}{*}{$\mathrm{K}^{+}$} & GC & $3( \pm 0,18)$ & $3,1( \pm 0,14)$ & $3,1( \pm 0,16)$ & $3,1( \pm 0,17)$ \\
\hline & $\mathrm{GI}$ & $2,9( \pm 0,38)$ & $3,3( \pm 0,74)^{b}$ & $2,8( \pm 0,32)$ & $2,8( \pm 0,44)$ \\
\hline \multirow{2}{*}{$\begin{array}{c}\mathrm{HCO}_{3} \\
(\mathrm{mmol} / \mathrm{L})\end{array}$} & GC & $26( \pm 2,01)$ & $26,4( \pm 1,52)$ & $26,2( \pm 1,76)$ & $26,1( \pm 1,86)$ \\
\hline & $\mathrm{Gl}$ & $26,2( \pm 1,63)$ & $20,6^{\mathrm{ab}}( \pm 2,53)$ & $22,8( \pm 3,12)^{b}$ & $25,3( \pm 2,65)$ \\
\hline $\mathrm{BE}$ & $\mathrm{GC}$ & $1,8( \pm 2,29)$ & $2,3( \pm 1,7)$ & $2( \pm 1,97)$ & $1,9( \pm 2,13)$ \\
\hline$(\mathrm{mmol} / \mathrm{L})$ & $\mathrm{GI}$ & $1,7( \pm 1,97)$ & $-4,8( \pm 3,26)^{a b}$ & $-2,3( \pm 3,95)^{b}$ & $0,9( \pm 3,03)$ \\
\hline \multirow{2}{*}{$\mathrm{SatO}_{2}$} & GC & $96( \pm 1,39)$ & $97( \pm 0,59)$ & $96( \pm 0,74)$ & $96( \pm 0,74)$ \\
\hline & $\mathrm{Gl}$ & $96( \pm 0,76)$ & $97( \pm 0,49)$ & $97( \pm 0,52)$ & $96( \pm 0,56)$ \\
\hline
\end{tabular}

a Diferença significativa entre grupos pelo teste $T$ pareado $(P<0,05)$.

b Difere de M0 do mesmo grupo, pelo teste Student Newman Keuls $(P<0,05)$.

\section{DISCUSSÃO}

A dor é responsável pelo estresse e pode levar a ansiedade (Clark et al. 1997). Estresse é um padrão de comportamento adaptativo, neuronal, endócrino, imunitário, que pode levar a alterações hematológicas e metabólicas voltadas para o restabelecimento da homeostase (Anderson \& Muir 2005).

Segundo Moberg (1996), estresse pode ser definido como a resposta biológica ou conjunto de reações obtidas quando um indivíduo percebe uma ameaça à sua homeostase. Esta ameaça pode ocorrer por vários motivos: fome, fadiga, lesão, temperatura ambiente extrema, ou até por fatores psicológicos, como contenção, manejo ou variação no manejo (Grandin 1997).

Surgiu no mercado um novo método de contenção fí- sica para bovinos, o Imobilizador Bovino, segundo o fabricante é um método de contenção seguro e não provoca estresse ao animal. Com base em observações clínicas com o uso deste equipamento, este projeto avaliou os níveis de cortisol, parâmetros cardiorrespiratórios e hemogasométricos com o objetivo de caracterizar a resposta do paciente frente à imobilização eletromagnética em bovinos.

Para não haver interferência do ciclo circadiano sobre os níveis sanguíneos de cortisol, este experimento foi sempre realizado durante o período matutino, como descrito anteriormente por Graf \& Seen (1999). Nos animais do grupo controle os parâmetros fisiológicos, níveis plasmáticos de cortisol e dados hemogasométricos mantiveram-se estáveis não indicando situação estressante ou 


\begin{abstract}
Quadro 2. Representação da resposta ao estímulo doloroso em percentagem de acordo com a escala préestabelecida, no grupo controle e imobilizador nos diferentes momentos. (0) não responde ao estímulo doloroso, (1)

resposta fraca ao estímulo doloroso, (2) resposta moderada ao estímulo doloroso, (3) resposta máxima ao estímulo doloroso (modificado da escala única descritiva
\end{abstract} (SDS) Holton et al. 1998)

\begin{tabular}{cccccc}
\hline \multirow{2}{*}{ Grupo } & Escala & \multicolumn{4}{c}{ Momentos } \\
\cline { 2 - 6 } & & M0 & M1 & M2 & M3 \\
\hline \multirow{3}{*}{ GC } & 0 & - & - & - & - \\
& 1 & - & - & - & - \\
& 2 & - & - & - & $16,66 \%$ \\
& 3 & $100 \%$ & $100 \%$ & $100 \%$ & $83,33 \%$ \\
GI & 1 & - & $100 \%$ ab & $16,66 \%$ & - \\
& 2 & - & - & $33,32 \%$ & $16,66 \%$ \\
& 3 & $100 \%$ & - & $16,66 \%$ & $33,32 \%$ \\
& & & - & $33,32 \%$ & $49,98 \%$
\end{tabular}

a Significativamente diferente do outro grupo, pelo teste de Wilcoxon $(\mathrm{P}<0,001) ;{ }^{b}$ difere de $\mathrm{MO}$ do mesmo grupo, pelo teste de Wilcoxon $(\mathrm{P}<0,001)$.

dolorosa, demonstrando que os mesmos foram manejados e condicionados ao ambiente de contenção de forma correta.

Nas variáveis $\mathrm{FC}, f$, PAM foi observado um aumento significativo nos animais do Gl logo após a utilização do imobilizador eletromagnético, permanecendo esses valores elevados até 1 hora após a utilização do mesmo, e também em relação ao GC, o que pode ser interpretado como estresse, ou alguma situação dolorosa sofrida pelos animais do $\mathrm{Gl}$, confirmando que o estresse pode ser avaliado por mudanças em parâmetros fisiológicos (Bonelli et al. 2008). Peers et al. (2002), observaram em seu experimento com castração e amputação de cauda através de anel de borracha em cordeiros, aumento significativo na FC, PAM e níveis de cortisol principalmente na primeira hora após os procedimentos, indicando que os animais passaram por um estímulo doloroso através desse método. Mellor et al. (2002), observaram em bovinos submetidos a descorna sem anestesia local, um aumento marcante nos níveis de cortisol, sendo que os níveis de cortisol diminuíram a valores próximos dos níveis do grupo controle com o bloqueio do nervo cornual. De forma semeIhante Kuchel et al. (1990), também encontraram aumento significativo na FC e PAM em ovinos conscientes submetidos à eletroimobilização.

Stafford et al. (2002), Mellor et al. (2002) e Obritzhauser et al. (1998) relatam em seus trabalhos que todos os métodos de castração levam a um aumento significativo no cortisol devido ao processo de dor e estresse. Taschke \& Folsch (1997) demonstraram que o procedimento de descorna traz estresse e é marcado por intenso aumento nos níveis de cortisol na saliva de vacas estudadas. Lay et al. (1992), encontraram após marcação dos animais com ferro quente, elevação da frequência cardíaca e das concentrações de cortisol, indicativos de sensação dolorosa nos animais.
Ainda quando utilizado o imobilizador eletromagnético ligado em uma potência onde o animal curve a coluna, conforme instrução do fabricante (Korth-Rfid 2009), foram observados em todos os animais do Gl mugidos e rigidez dos músculos voluntários dos membros, o que associado ao aumento nas variáveis $\mathrm{FC}, f$ e PAM nos animais desse grupo, sugere uma situação dolorosa ou estressante.

Alterações comportamentais, como vocalização excessiva (Weary \& Fraser 1995, Weary et al. 1998), alteração de postura e da atividade locomotora (Ley et al. 1991, McGlone et al. 1993, Molony \& Kent 1997, Whay 1997, Thornton \& Waterman-Pearson 1999), sugerem um status de mal-estar aos animais.

Segundo Sneddon \& Gentle (2001), o problema com a interpretação desses parâmetros é que o estresse também provoca a liberação de cortisol e aumento dos batimentos cardíacos e pode argumentar-se que o animal pode estar tanto estressado quanto com dor.

O nível sérico de cortisol é amplamente reconhecido como um marcador geral de estresse, e tem sido largamente utilizado para identificar o bem estar de animais de fazenda durante procedimentos agropecuários (Fulkerson et al. 1982, Shutt et al. 1988, Hargreaves \& Hutson 1990, Graham et al. 1997, Sutherland et al. 2000).

Neste estudo, os níveis plasmáticos de cortisol no GI permaneceram aumentados até 1 hora após a retirada da sonda imobilizadora quando comparados com os valores basais e com o GC. Bonelli et al. (2008) em seu experimento, com diferentes métodos de castração, observou que o cortisol sérico não retornou aos níveis de pré-tratamento após 180 minutos em nenhum grupo, exceto no grupo controle. Neste experimento embora não realizado procedimento invasivo conforme o realizado por Bonelli et al. (2008) o simples fato do uso do imobilizador eletromagnético ligado produziu aumento significativo nos níveis de cortisol, sugerindo que o animal pode estar estressado ou com dor, concordando com os achados de Sneddon \& Gentle (2001).

O aumento dos níveis de cortisol e a liberação de catecolaminas elevam o débito cardíaco e a pressão arterial, aumentando o fornecimento de substratos aos tecidos que são essenciais para a defesa imediata do organismo (Genuth 2000), o que concorda com o aumento dos níveis plasmáticos de cortisol e da PAM nos animais do Gl em nossos resultados.

Com relação aos dados hemogasométricos, os valores de $\mathrm{pH}$ apresentaram-se dentro dos valores aceitáveis, porém foi observado que no Gl logo após a utilização do imobilizador houve uma redução no $\mathrm{pH}$ retornando uma hora após a valores próximos dos basais, fato que não ocorreu no GC. A produção excessiva de íons $\mathrm{H}^{+}$, com consequente queda no $\mathrm{pH}$, pode ser causada por três situações diferentes: pela geração de ácido láctico ou demais ácidos, menor excreção de íons $\mathrm{H}^{+}$do organismo e perda de bicarbonato (Ortolani 2003). A redução no pH pode ter ocorrido no momento da utilização do imobilizador 
eletromagnético, devido ao estímulo estressante e doloroso provocar uma vasoconstrição periférica, e com isso a formação de lactato reduzindo os valores de $\mathrm{pH}$ nos animais desse grupo. Tal fato foi, também, observado por Kuchel et al. (1990), em ovinos submetidos à eletroimobilização, os quais apresentaram um aumento no fluxo de lactato com consequente redução nos valores de $\mathrm{pH}$, e por Thorburn et al. (1990) na mesma espécie.

$\mathrm{A} \mathrm{PaCO}_{2}$ nos animais do $\mathrm{Gl}$ apresentaram uma redução significativa em relação ao momento basal e em relação ao GC principalmente após o uso imediato do imobilizador eletromagnético, essa redução da $\mathrm{PaCO}_{2}$ associada ao aumento da $f$ demonstram que os animais do Gl entraram em alcalose respiratória após a utilização do imobilizador. Kuchel et al. (1990), observaram resultados semelhantes, com redução nos valores de $\mathrm{PaCO}_{2}$ em ovinos submetidos à eletroimobilização. Almosny (2003) sugere dentre diversas causas de alcalose respiratória (hiperventilação) situações de ansiedade, medo e dor. Segundo Muir \& Moraes (1996), observa-se redução de $0,55 \mathrm{mEq} / \mathrm{L}$ de bicarbonato para cada $\mathrm{mmHg}$ de decréscimo de $\mathrm{PaCO}_{2}$, fato que coincide com os dados encontrados neste trabalho, onde encontramos redução nos

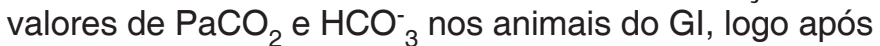
a utilização do imobilizador.

Os níveis de $\mathrm{HCO}_{3}^{-}$e $\mathrm{BE}$ no $\mathrm{Gl}$ após a utilização do imobilizador diminuíram significativamente, o que associado ao aumento nos níveis de $\mathrm{K}^{+}$, nos indica que essas alterações podem ter ocorrido na tentativa dos animais desse grupo em tamponar a redução no $\mathrm{pH}$. O excesso de base (BE) indica indiretamente a quantidade de tampões existentes dentro do sangue, numa dada temperatura e pressão de dióxido de carbono (Ortolani 2003). $\mathrm{O} \mathrm{HCO}_{3}^{-}$é responsável por mais de $50 \%$ da capacidade tampão extraceIular (Luna 2002). Segundo DiBartola \& Morais (2007), em geral a acidose metabólica está associada a transferência de íons $\mathrm{K}^{+}$do fluido intracelular para o fluido extracelular. $\mathrm{O}$ principal mecanismo envolvido na hipercalemia que ocorre na acidose é a troca de íons $\mathrm{H}^{+}$extracelular, pelo $\mathrm{K}^{+}$ intracelular (Carmona \& Mendonça 2002). Estudos em animais indicam que acidose metabólica aguda está associada ao aumento do $0,6 \mathrm{mEq} / \mathrm{L}$ de $\mathrm{K}^{+}$para cada diminuição de 0,1U no pH (Schwartz et al. 1957).

Com relação à resposta ao estímulo, os animais do GI apresentaram resposta negativa no momento da estimulação, quando comparado ao GC. Com a utilização da sonda imobilizadora ligada, $100 \%$ dos animais não apresentaram resposta ao estímulo doloroso através do pinçamento da vulva. Apesar desse resultado o aumento nos parâmetros fisiológicos FC, $f$, PAM e níveis plasmáticos de cortisol associado a alterações comportamentais como vocalizações e contração dos músculos voluntários dos membros frente à imobilização eletromagnética sugerem que os animais sofreram um estímulo estressante ou doloroso.

Comprovando a afirmação de Fitzpatrick et al. (2006), onde os animais de produção, particularmente ruminan- tes, têm comportamento relativamente tranquilo, onde é possível que os mesmos não demonstrem sinais de estresse e dor de modo claro, levando a erros de avaliação pelos observadores. Portanto, métodos com o objetivo de mensurar dor e bem-estar em animais de produção precisam ser muito bem estudados, para que se obtenham resultados fidedignos de tais avaliações.

\section{CONCLUSÃO}

De acordo com a avaliação de parâmetros cardiorrespiratórios, níveis plasmáticos de cortisol e dados hemogasométricos, podemos concluir que o imobilizador eletromagnético deve ser utilizado apenas para imobilização física, não sendo possível realizar procedimentos cirúrgicos, pois o mesmo não oferece nenhum suporte anestésico ao animal.

\section{REFERÊNCIAS}

Anderson D.E. \& Muir W.W. 2005. Pain management in ruminants. Vet. Clin. Food Anim. 21:19-31.

Almosny N. 2003. Equilíbrio ácido-básico em medicina veterinária, p.516. In: González F.H.D. \& Campos R. (Eds), Anais do 1ํㅗำósio de Patologia Clínica Veterinária da Região Sul do Brasil. Gráfica da Universidade Federal do Rio Grande do Sul, Porto Alegre.

Bonelli P., Dimauro C., Pau S., Dattena M., Mollica A. \& Nicolusi P.S. 2008. Stress responses in lambs castrated with three different methods. Ital. J. Anim. Sci. 7:207-217.

Carmona M.J.C. \& Mendonça M.R.F. 2002. Distúrbios hidroeletrolíticos e anestesia, p.130-139. In: Fantoni D.T. \& Cortopassi S.R.G. (Eds), Anestesia em Cães e Gatos. Roca, São Paulo.

Clark J.D., Rager D.R. \& Calpin J.P. 1997. Animal well-being: I. General considerations. Lab. Anim. Sci. 47:564-570.

DiBartola P.S. \& Morais H.A. 2007. Distúrbios Relacionados ao Potássio: Hipo e Hipercalemia, p.87-114. In: DiBartola S. (Ed.), Anormalidades de Fluidos, Eletrólitos e Equilíbrio Ácido-Basico. 3ª ed. Roca, São Paulo.

Fantoni D.T. \& Mastrocinque S. 2002. Fisiopatologia da dor, p.323-334. In: Fantoni D.T. \& Cortopassi S.R.G. (Eds), Anestesia em Cães e Gatos. Roca, São Paulo.

Fitzpatrick J., Scott M. \& Nolan A. 2006. Assessment of pain and welfare in sheep. Small Rum. Res. 62:55-61.

Fulkerson W.J. \& Jamieson P.J. 1982. Pattern of cortisol release in sheep following administration of ACTH or imposition of various stressor agents. Aust. J. Biol. Sci. 35:215-222.

Genuth S.M. 2000. Supra-renais, p.877-909. In: Berne R.M. \& Levy M.N. (Eds), Fisiologia. Guanabara Koogan, Rio de Janeiro.

Graf B. \& Senn M. 1999. Behavioural and physiological responses of calves to dehorning by heat cauterization with or without local anaesthesia. Appl. Anim. Behav. Sci. 62:153-171.

Graham M.J., Kent J.E. \& Molony V. 1997. Effects of four analgesic treatments on the behavioural and cortisol responses of 3-week-old lambs to tail docking. Vet. J. 153:87-97.

Grandin T. 1997. Assesment of stress during handling and transport. J. Anim. Sci. 75:249-257.

Hargreaves A.L. \& Hutson G.D. 1990. Changes in heart rate, plasma cortisol and haematocrit of sheep during a shearing procedure. Appl. Anim. Behav. Sci. 26:91-101.

Holton L.L., Scott E.M., Nolan A.M., Reid J.W. \& Flaherty D. 1998. Com-parison of three methods used for assessment of pain in dog. J. Am. Vet. Med. Assoc. 212:795-796. 
Korth-Rfid 2009. Imoboi, imobilização eletrônica segura. São Carlos, SP. Disponível em: http://www.animalltag.com.br/produtos.php? prodid=14 (Acesso em 10 de Janeiro de 2009).

Kuchel T.R., Mather L.E., Runciman W.B. \& Carapetis R.J. 1990. Physiological and biochemical consequences of eletroimobilisation in concious sheep. Aust. Vet. J. 67:33-38.

Lay D.C.Jr, Friend T.H., Bowers C.L., Grissom K.K. \& Jenkins O.C. 1992. A comparative physiological and behavioral study of freeze and hot-iron branding using dairy cows. J. Anim. Sci. 70(4):1121-1125.

Ley S.J., Livingston A. \& Waterman A.E. 1991. Effects of chronic lameness on the concentrations of cortisol, prolactin and vasopressin in the plasma of sheep. Vet. Rec. 129(3):45-47.

Luna S.P.L 2002. Equilíbrio ácido-básico, p. 120-129. In: Fantoni D.T. \& Cortopassi S.R.G. (Eds), Anestesia em Cães e Gatos. Roca, São Paulo.

McGlone J.J., Nicholson R.I., Hellmnan J.M. \& Herzog D.N. 1993. The development of pain in young pigs associated with castration and attempts to prevent castration induced behavioral changes. J. Anim. Sci. 71(6):1441-1446.

Mellor D.J., Stafford K.J., Todd S.E., Lowe T.E., Gregory N.G., Bruce R.A. \& Ward R.N. 2002. A comparison of catecholamine and cortisol responses of young lambs and calves to painful husbandry procedures. Aust. Vet. J. 80(4):228-233.

Moberg G.P. 1996. Suffering from stress: An approach for evaluating the welfare of an animal, p.46-49 In: Sandoe P. \& Hurnik T. (Eds), Proceedings of Welfare of Domestic Animals Concepts, Theories and Methods of Measurement. Acta Agriculturae Scandinavica, Sect. A, Anim. Sci.

Molony V. \& Kent J.E. 1997. Assessment of acute pain in farm animals using behavioral and physiological measurements. J. Anim. Sci. 75(1):266-272.

Muir W.W. \& de Moraes H.S.A. 1996. Acid-base balance: Traditional and modified approaches, p.558-571. In: Thurmon C.E., Tranquilli W.J. \& Benson G.J (Eds). Lumb \& Jone's Veterinary Anesthesia. Williams and Wilkins, Baltimore.

Obritzhauser W., Deutz A. \& Kofer J. 1998. Comparison of two castration methods in cattle: Plasma cortisol levels, leukocyte count and behavioral changes. Tierärztl. Prax. G, Grosstiere Nutztiere, 26(3):119-126.

Ortolani E.L. 2003. Diagnóstico e tratamento de alterações ácido-básicas em ruminantes, p. 17-28. In: González F.H.D. \& Campos R. (Eds), Anais do $1^{\circ}$ Simpósio de Patologia Clínica Veterinária da Região Sul do Brasil. Gráfica da Universidade Federal do Rio Grande do Sul, Porto Alegre.
Paddleford R.R. 2001. Analgesia e controle da dor, p. 264-285. In: Ibid. (Ed.), Manual de Anestesia em Pequenos Animais. $2^{\mathrm{a}}$ ed. Roca, São Paulo.

Peers A., Mellor D.J., Wintour E.M. \& Dodic M. 2002. Blood pressure, heart rate, hormonal and other acute responses to rubber ring castration and tail docking of lambs. N. Z. Vet. J. 50(2):56-62.

Schwartz W.B., Orning K.J. \& Porter R. 1957. The internal distribution of hydrogen ions with varying degrees of metabolic acidosis. J. Clin. Invest. 36:373.

Shutt D.A., Fell L.R., Connell R. \& Bell A.K. 1988. Stress responses in lambs docked and castrated surgically or by the application of rubber rings. Aust. Vet. J. 65:5-7.

Sneddon L.U. \& Gentle M.J. 2001. Pain in Farm Animals. Disponível em: http://www.agriculture.de/acms1/con f6/ws5apain.html (Acesso em 8 de julho de 2008).

Sutherland M.A., Stafford K.J., Mellor D.J., Gregory N.G., Bruce R.A. \& Ward R.N. 2000. Acute cortisol responses and wound healing in lambs after rings castration plus tail docking with or without the application of a castration clamp to the scrotum. Aust. Vet. J. 78:402-405.

Stafford K.J., Mellor D.J., Todd S.E., Bruce R.A. \& Ward R.N. 2002. Effects of local anaesthesia or local anaesthesia plus a non-steroidal anti-inflammatory drug on the acute cortisol response of calves to five different methods of castration. Res. Vet. Sci. 73(1):61-70.

Stöber M. 1990. Manejo de Bovinos, p.1-44. In: Dirksen G., Gründer H. \& Stöber M. (Eds), Rosenberger's Exame Clínico de Bovinos. Guanabara Koogan, Rio de Janeiro.

Taschke A.C \& Folsch D.W. 1997. Ethological, physiological and histological aspects of pain and stress in cattle when being dehorned. Tierärztl. Praxis 25(1):19-27.

Thorburn G.D., Jephcott E.H., Lynn R.D. \& Mcmillen I.C. 1990. Effects of electroimmobilisation on blood gas and $\mathrm{pH}$ status in sheep. Res. Vet. Sci. 48(3):314-320.

Thornton P.D. \& Waterman-Pearson A.E. 1999. Quantification of the pain and distress responses to castration in young lambs. Res. Vet. Sci. 66(2):107-118.

Wall P.D. 1992. Defining "Pain in Animals", p.63-79. In: Short C.E. \& Poznak A.V. (Eds), Animal Pain. Churchill Livingstone, New York.

Weary D.M., Braithwaite L.A. \& Fraser D. 1998. Vocal responses to pain in piglets. Appl. Anim. Behav. Sci. 56(2/4):161-172.

Weary D.M. \& Fraser D. 1995. Signalling need: Costly signals and animal welfare assessment. Appl. Anim. Behav. Sci. 44(2/4):159-169.

Whay H.R. 1997. Pain in the lame cow. Vet. Rec. 50(10):603-609. 